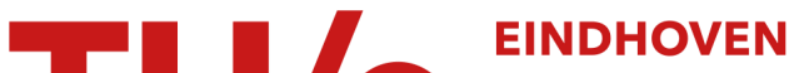 UNIVERSITY OF TECHNOLOGY
}

\section{On the relationship between uniform and recurrent nonuniform discrete-time sampling schemes}

\section{Citation for published version (APA):}

Sommen, P. C. W., \& Janse, K. (2008). On the relationship between uniform and recurrent nonuniform discretetime sampling schemes. IEEE Transactions on Signal Processing, 56(10), 5147-5156.

https://doi.org/10.1109/TSP.2008.928695

DOI:

10.1109/TSP.2008.928695

Document status and date:

Published: 01/01/2008

\section{Document Version:}

Publisher's PDF, also known as Version of Record (includes final page, issue and volume numbers)

\section{Please check the document version of this publication:}

- A submitted manuscript is the version of the article upon submission and before peer-review. There can be important differences between the submitted version and the official published version of record. People interested in the research are advised to contact the author for the final version of the publication, or visit the $\mathrm{DOI}$ to the publisher's website.

- The final author version and the galley proof are versions of the publication after peer review.

- The final published version features the final layout of the paper including the volume, issue and page numbers.

Link to publication

\section{General rights}

Copyright and moral rights for the publications made accessible in the public portal are retained by the authors and/or other copyright owners and it is a condition of accessing publications that users recognise and abide by the legal requirements associated with these rights.

- Users may download and print one copy of any publication from the public portal for the purpose of private study or research.

- You may not further distribute the material or use it for any profit-making activity or commercial gain

- You may freely distribute the URL identifying the publication in the public portal.

If the publication is distributed under the terms of Article 25fa of the Dutch Copyright Act, indicated by the "Taverne" license above, please follow below link for the End User Agreement:

www.tue.nl/taverne

Take down policy

If you believe that this document breaches copyright please contact us at:

openaccess@tue.nl

providing details and we will investigate your claim. 


\title{
On the Relationship Between Uniform and Recurrent Nonuniform Discrete-Time Sampling Schemes
}

\author{
Piet Sommen and Kees Janse
}

\begin{abstract}
Recurrent nonuniform discrete-time signal samples can be regarded as a combination of $K$ mutual delayed sequences of uniform discrete-time signal samples taken at one $K$ th of the Nyquist sampling rate. This paper introduces a new alternative discrete-time analysis model of the recurrent nonuniform sampling scenario. This model can be described by the analysis part of a uniform discrete Fourier transfrom (DFT) modulated filterbank from which the $K$ uniformly distributed and downsampled frequency bands are mixed in a very specific way. This description gives a clear relationship between uniform and recurrent nonuniform discrete-time sampling schemes. A side benefit of this model is an efficient structure with which one can reconstruct uniform discrete-time Nyquist signal samples from recurrent nonuniform samples with known mutual delays between the nonuniform distributed samples. This reconstruction structure can be viewed as a natural extension of the synthesis part of an uniform DFT modulated filterbank.
\end{abstract}

Index Terms-Discrete Fourier transfrom (DFT), nonuniform DFT, recurrent nonuniform sampling, sampling, uniform DFT modulated filterbank.

\section{INTRODUCTION}

$\mathbf{T}$ HE most common form of sampling in discrete-time signal processing is uniform sampling. However, there are a variety of applications in which the signals are sampled differently. Some of these applications are mentioned and discussed in [4], [8], and [9]. In the current paper, we do not focus on one specific application. For this reason we will use a very high-speed analog-to-digital (A/D) convertor as a guiding example throughout this paper. Such an A/D convertor is composed of more than one, say $K$, parallel low-speed A/D convertors which are combined in a time interleaved way. It is a well-known fact that this is a power-efficient technique that can be used to increase the maximum sample rate [8]. When the analog input signal $x(t)$ is bandlimited with maximum frequency $\left|f_{\max }\right|<1 / 2 T_{0}$, where $1 / T_{0}$ is the Nyquist sampling rate, each of the $K$ low speed A/D convertors is sampled at a factor $1 / K$ of the desired Nyquist sampling rate. At the reconstruction part of the system, the undersampled signals of the $K$ branches are combined to construct the uniform discrete-time

Manuscript received November 8, 2007; revised June 4, 2008. First published July 14, 2008; current version published September 17, 2008. The associate editor coordinating the review of this manuscript and approving it for publication was Dr. Thierry Blu.

P. Sommen is with the Signal Processing Systems group, Electrical Engineering Department, Technische Universiteit Eindhoven, The Netherlands.

K. Janse is with the Digital Signal Processing Group of Philips Research Laboratories, Eindhoven, The Netherlands.

Digital Object Identifier 10.1109/TSP.2008.928695 signal samples $x\left[n T_{0}\right]$ at the Nyquist sampling rate. Since each of the $K$ low-speed A/D convertors sample the original signal $x(t)$ at another moment, there will be a time-offset (delay) between the low-speed A/D convertors. In practice, it is extremely difficult to uniformly distribute these delays in a period of $K \cdot T_{0}$ [s]. The result is that time interleaving the $K$ signal samples make up a sequence of recurrent nonuniform signal samples.

Reconstruction from nonuniform signal samples is considerably more complex than reconstruction from uniform signal samples and is investigated by many researchers. The main focus of [1]-[14] is on the theoretical background and the reconstruction schemes are computationally demanding. Practical, efficient reconstruction algorithms are still required. Papoulis [14] showed how Shannon's sampling theorem can be generalized to allow a bandlimited signal to be recovered from uniformly spaced samples of the outputs of $K$ different linear time-invariant filters with the signal as their input sampled at one- $K$ th of the Nyquist rate. This result was extended in [17] without the bandlimitation constraint, while extensions to more dimensions can be found in [3] and [7]. A general sampling theory for nonideal acquisition devices is derived in [16]. The emphasis of [18] is on nonbandlimited signals, pointwise stability of reconstruction, and reconstruction from nonuniform samples. A survey that provides a unified framework for uniform and nonuniform sampling and reconstruction is given in [1].

In contrast to the bandlimited case that is studied in the current paper, [20] and [6] study the problem of recurrent nonuniform sampling and its reconstruction for multiband signals. These signals do not occupy the whole frequency band and uniform (Nyquist) sampling can become very redundant. Furthermore in [20], the $K$ different delays are modeled as fractional delays $c_{k} / L$, with both $c_{k}$ and $L$ integer numbers. Depending on the value of the delay, $L$ can become very large. Above that, this number $L$ is used as upsample factor in each of the $K$ branches of the reconstruction scheme. The current paper does not restrict the delays to fractional delays and the upsamplers in the $K$ branches of the reconstruction scheme are factor $K$ upsamplers.

Papers [4] and [10] introduce a filterbank interpretation of various sampling strategies. In case of recurrent nonuniform sampling, the first step of the reconstruction scheme in these papers is to apply in each of the $K$ parallel branches an upsampler of factor $K$ and the second step is to filter each of these $K$ resulting upsampled signals with different filters. In [4], these filters are multilevel filters, that is these filters are piecewise constant over a frequency interval $2 \pi / K$. In the current paper, a 
new alternative model is introduced in such a way that there is a simple mutual relation between the different filters of the $K$ branches. This mutual relation can be used to switch the order of the upsamplers and the filters, resulting in a realization scheme with improved efficiency.

Another approach that uses discrete Fourier transfroms (DFTs) instead of filterbanks can be found in [9]. Here, the reconstruction of the uniform signal samples is done by first calculating, at a uniform frequency grid with resolution $2 \pi / K$, a DFT of the nonuniform distributed signal samples. It is shown how these values are related to the continuous-time Fourier transform. The inverse of this relation is used to produce $K$ different values of the continuous-time Fourier transform at a uniform grid with resolution $2 \pi / K$. Transforming these $K$ values back to discrete-time domain, with an inverse DFT, results in a set of $K$ reconstructed uniform signal samples. In order to construct a whole sequence of uniform signal samples, this procedure is repeated. Because of the usage of the DFTs, the described results are only valid for periodic bandlimited signals. Similar approaches are given in [12], [15] and [19]. The last two papers also derive different techniques to find the delays if these are not known in advance. A combinatorial solution of this problem can be found in [12], while [19] describes a technique for real-valued delays.

Finally, the authors of [8] describe techniques for correcting sample-time errors in a time interleaved A/D convertor. They also give an adaptive scheme to update the unknown delays. However they restrict themselves to $K=2$ branches and small delays (sample-time errors).

The main goal of the current paper is to clarify the relationship between uniform and recurrent nonuniform sampling schemes of bandlimited signals. For this purpose a new alternative discrete-time analysis model of the recurrent nonuniform sampling scenario is introduced. This model can be described by the analysis part of a uniform DFT modulated filterbank from which the $K$ uniformly distributed and downsampled frequency bands are mixed in a very specific way. This description gives a clear relationship between uniform and recurrent nonuniform discrete-time sampling schemes. A side benefit of this relationship is an efficient reconstruction structure with which one can reconstruct uniform discrete-time Nyquist signal samples from recurrent nonuniform samples from which the mutual delays between the nonuniform distributed samples are known. This reconstruction structure can be viewed as a natural extension of the synthesis part of an uniform DFT modulated filterbank [13].

The organization of this paper is as follows. In Section II we formulate the recurrent nonuniform sampling scenario and give a discrete-time model for this scenario. A new alternative discrete-time model and its description are derived in Section III. This alternative model can be represented by a uniform DFT modulated filterbank as will be explained in Section IV. This representation leads in Section $\mathrm{V}$ to an efficient DFT modulated filterbank realization for the reconstruction of uniform discrete-time Nyquist signal samples from recurrent nonuniform samples. In Section VI we discuss simulation results and the paper is finished with conclusions in Section VII.

Finally we note here that in practice noncausal results can always be obtained by applying an appropriate delay. For this

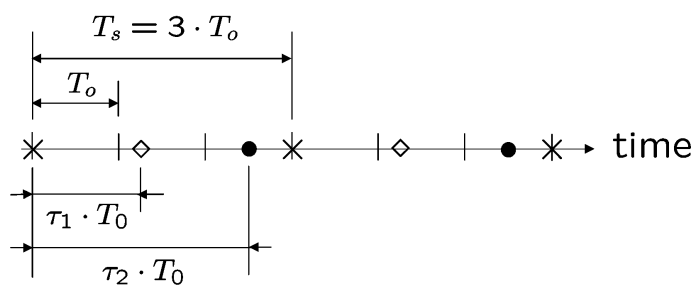

Fig. 1. Example of recurrent nonuniform signal samples for $K=3$.

reason we will not be concerned with causality. Furthermore we will use lower case characters for the representation of signals in time domain and upper case characters for frequency domain representation. Underlined boldface characters are used for vectors, boldface characters for matrices, $\operatorname{diag}\{\cdot\}$ is a diagonal matrix, while $(\cdot)^{t}$ denotes the transpose of a vector.

\section{RECURRENT NONUNIFORM SAMPLING}

\section{A. Sampling Scheme}

In this paper, we focus on the recurrent nonuniform sampling scheme. In this form of sampling, the sampling points are divided into groups of $K$ points each. The groups have a recurrence period, which is denoted by $T_{s}$, that is equal to $K$ times the Nyquist period $T_{0}$. Each period consists of $K$ nonuniform sampling points. Denoting the sampling points in one period $T_{s}$ by $\tau_{k} \cdot T_{0}$, for $k=0,1, \ldots, K-1$, the complete set of sampling points for $n \in(-\infty, \infty)$ is given by

$$
\tau_{k} \cdot T_{0}+n \cdot T_{s} \text { with } 0 \leq \tau_{k}<K
$$

where $T_{s}=K \cdot T_{0}$. We will assume throughout this paper that all delays are different, thus $\tau_{p} \neq \tau_{q}$ for $p \neq q$. Furthermore, without loss of generality, we use $\tau_{0}=0$ and $\tau_{p}<\tau_{q}$ for $p<q$. It is shown in [14] that the original bandlimited analog signal $x(t)$ is uniquely determined by the sampling points as defined in (1). From this equation and the assumptions, it follows straightforward that for $\tau_{k}=k$, with $k=0,1, \ldots, K-1$, the sequence of recurrent nonuniform samples reduces to a sequence of uniform samples. It is finally noted here that recurrent nonuniform samples can be regarded as a combination of $K$ sequences of uniform samples taken at one $K$ th of the Nyquist rate.

Example 1: An example of a recurrent nonuniform sampling distribution for the case $K=3$ is depicted in Fig. 1. This figure shows a time axis on which the small vertical lines have a distance of $T_{0}$ [s], the Nyquist period. With $K=3$ the undersampled period equals $T_{s}=3 \cdot T_{0}$ [s]. Within each undersampled period of $T_{s}$ [s] we have $K=3$ samples. The first sample point in each undersampled period is denoted with a $\times$ at $\tau_{0}=0$ [s]. The second sample point, denoted with $\mathrm{a} \diamond$, has a delay of $\tau_{1} \cdot T_{0}[\mathrm{~s}]$ with respect to the first sample point. Finally, the third sample point, denoted with a $\bullet$, has a delay of $\tau_{2} \cdot T_{0}$ [s] with respect to the first one.

\section{B. Sampling Scenario}

A recurrent nonuniform sampling scenario that covers a wide range of applications is sketched in Fig. 2. In this figure the uniformly distributed discrete-time signal samples $x\left[n T_{0}\right]=\left.x(t)\right|_{t=n T_{0}}$ are reconstructed by using $K$ parallel $\mathrm{A} / \mathrm{D}$ converters. Each of these convertors is operating at a 


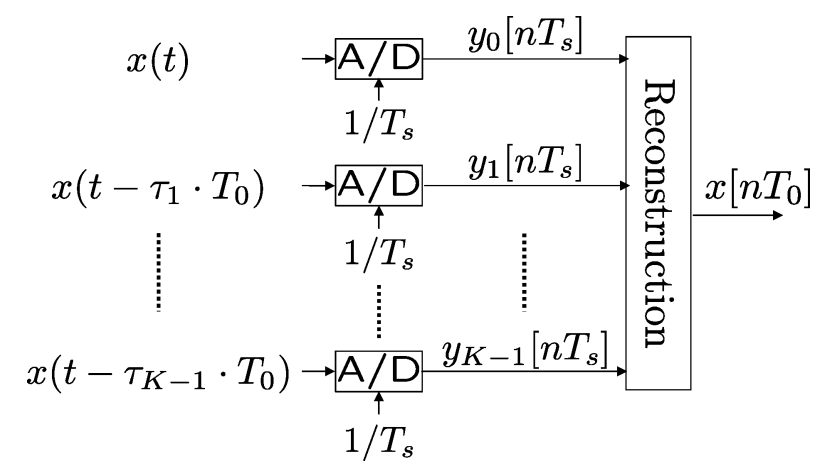

Fig. 2. Recurrent nonuniform sampling scenario with $K$ parallel A/D converters, each operating at a lower rate than the Nyquist rate $1 / T_{s}=1 /\left(K \cdot T_{0}\right)$, and the reconstruction of the uniformly distributed discrete-time signal samples $x\left[n T_{0}\right]=\left.x(t)\right|_{t=n T_{0}}$.

lower rate than the Nyquist rate $1 / T_{s}=1 /\left(K \cdot T_{0}\right)$ such that the average overall sampling rate is equal to the Nyquist rate $1 / T_{0}$. Without loss of generality, we will assume throughout this paper that the continuous-time signal $x(t)$ and its delayed versions $x\left(t-\tau_{k} \cdot T_{0}\right)$, for $k=0,1, \ldots, K-1$, are real valued baseband signals. Furthermore, we will assume that $x(t)$ is bandlimited to $\left|f_{\max }\right|<1 / 2 T_{0}$.

\section{Discrete-Time Model}

We will make our further derivation completely in the discrete-time domain. For this we need a discrete-time model of the recurrent nonuniform sampling scenario as depicted in the left-hand side (LHS) of Fig. 2. In order to derive this discretetime model the first step is to model each of the $K$ undersampled A/D converters by one A/D converter that is sampled at Nyquist rate $1 / T_{0}$ followed by a downsampler of a factor $K$. The second step is to model each of the analog delays in the discrete-time domain. The frequency response function of each of the analog delays is given by $\mathrm{e}^{-\mathrm{j} \tau_{k} \cdot T_{0} \omega}$ with $\omega=2 \pi f$ and $f$ the analog frequency $[\mathrm{Hz}]$. Since the amplitude characteristic of this function equals 1 for all frequencies, we will neglect it throughout this paper. A sketch of the analog phase characteristic is depicted in the LHS of Fig. 3. Because of the bandlimited character of $x\left(t-\tau_{k} \cdot T_{0}\right)$, the frequency response function of the discrete-time delay can be modeled by the periodic function $\Delta_{k}\left(\mathrm{e}^{\mathrm{j} \theta}\right)=\mathrm{e}^{-\mathrm{j} \tau_{k} \theta}$, where $\theta=\omega \cdot T_{0}$ is the relative discrete-time frequency with period $2 \pi$. The discrete-time phase function $\Phi\{\cdot\}$ of $\Delta_{k}\left(\mathrm{e}^{\mathrm{j} \theta}\right)$ is shown at the right-hand side (RHS) of Fig. 3. In this figure, we have chosen the fundamental interval (FI) as $-\pi<\theta \leq \pi$. In this FI the phase of the discrete-time delay is given by the following linear function of $\theta$ :

$$
\Phi\left\{\Delta_{k}\left(\mathrm{e}^{\mathrm{j} \theta}\right)\right\}=-\tau_{k} \cdot \theta
$$

Without loss of generality we will neglect, both in the equations and the figures, a possible phase jump from $-\pi$ to $\pi$. Further on in this paper it will appear that the phase jump from $-\tau_{k} \cdot \pi$ to $\tau_{k} \cdot \pi$ at the edge of the FI needs extra attention since it plays an important role in some basic equations of the derivation. For the uniform sampling case, thus when $\tau_{k}=k$, this phase jump equals $k \cdot 2 \pi$ and can be neglected. However for the nonuniform
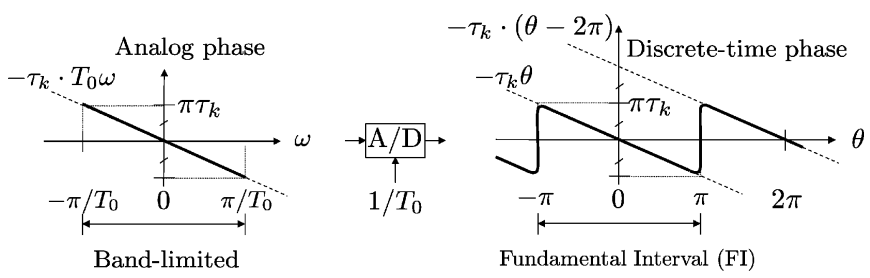

Fig. 3. Phase relation between analog and discrete-time delay.

sampling case $\tau_{k}$ does not need to be an integer number and thus this phase jump equals $\tau_{k} \cdot 2 \pi$ and can not be neglected.

These two steps result in the discrete-time model of the recurrent nonuniform sampling scenario as depicted at the RHS of Fig. 4.

In order to clarify the relationship between recurrent nonuniform signal samples and uniform signal samples we will first introduce in this paper an alternative discrete-time model of the recurrent nonuniform sampling scenario.

\section{Alternative DisCRETE-TIME MODEL}

\section{A. Description of Alternative Model of One Branch}

To make our further derivation, we will first focus here on one branch $k$ and introduce an alternative model for this branch. For this reason, we will skip the index $k$ here.

The discrete-time model of one branch of the nonuniform sampling scenario is depicted in Fig. 5. In this figure, the discrete-time input signal samples $x\left[n T_{0}\right]$ are samples at Nyquist rate $1 / T_{0}$. These signal samples are delayed by the discrete-time delay, from which the frequency response function in the FI is given by $\Delta\left(\mathrm{e}^{\mathrm{j} \theta}\right)=\mathrm{e}^{-\mathrm{j} \tau \theta}$. The resulting signal samples are downsampled by a factor $K$ leading to the output signal samples $y\left[n T_{s}\right]$, with $T_{s}=K \cdot T_{0}$.

With the twiddle factor defined as $W_{K}=\mathrm{e}^{-\mathrm{j}(2 \pi / K)}$ and using the standard expression [13] for a downsampling operator $\downarrow K$, we obtain the following general expression in the frequency domain for the discrete-time output signal samples $y\left[n T_{s}\right]:$

$$
Y\left(\mathrm{e}^{\mathrm{j} \theta}\right)=\frac{1}{K} \sum_{p=0}^{K-1} \Delta\left(\mathrm{e}^{\mathrm{j} \theta / K} \cdot W_{K}^{-p}\right) \cdot X\left(\mathrm{e}^{\mathrm{j} \theta / K} \cdot W_{K}^{-p}\right) .
$$

For integer values of $\tau$, this equation reduces to

$$
Y\left(\mathrm{e}^{\mathrm{j} \theta}\right)=\frac{1}{K} \cdot \mathrm{e}^{-\mathrm{j} \tau \theta / K} \sum_{p=0}^{K-1} W_{K}^{p \tau} \cdot X\left(\mathrm{e}^{\mathrm{j} \theta / K} W_{K}^{-p}\right) .
$$

Thus in this case the output signal samples of Fig. 5 can be viewed as a time aligned linear combination of $K$ uniform frequency bands of the input signal samples. This simple relationship does not hold for noninteger values of $\tau$ since we need to take care of the phase jump, as described in the previous section and depicted in Fig. 3. This point of attention can be verified by the following simple example.

Example 2: In this example we use $T_{0}=1, x[n]=\delta[n]$, $K=2$ and the delay $\tau$ can be any noninteger number. With these parameters we can calculate on the one hand, directly from the model as depicted in Fig. 5, an expression for the output 

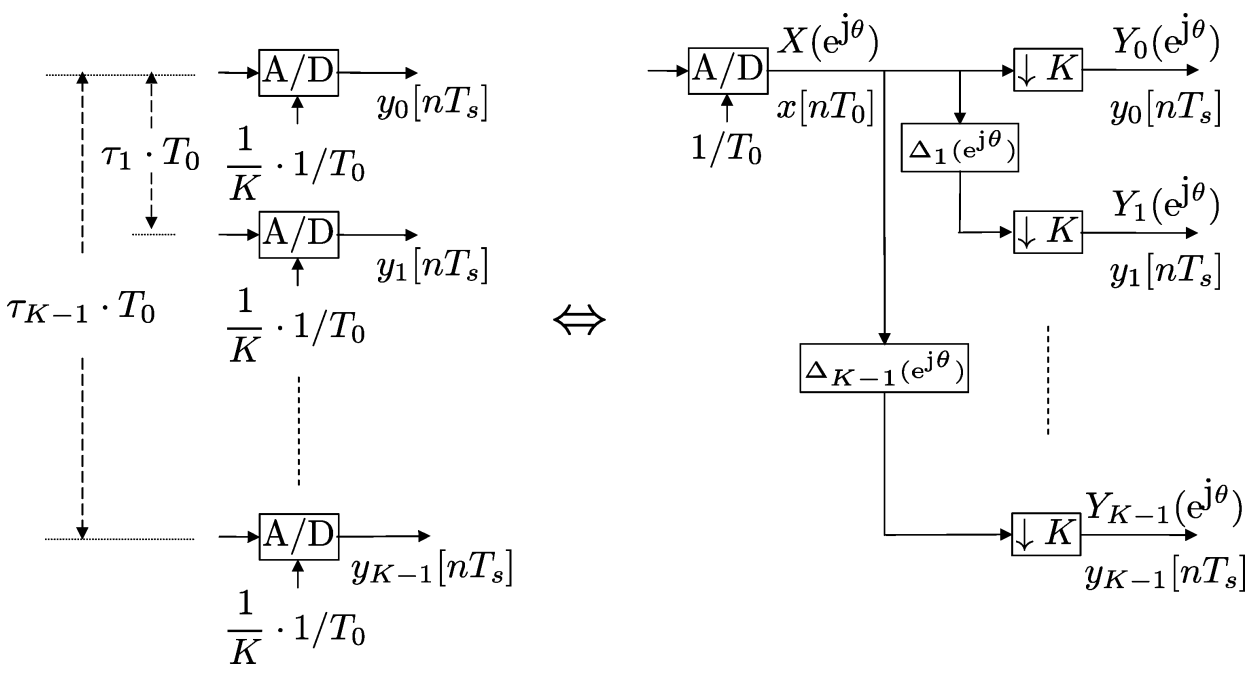

Fig. 4. Recurrent nonuniform sampling scenario. LHS: $K$ undersampled parallel A/D convertors. RHS: Discrete-time model.

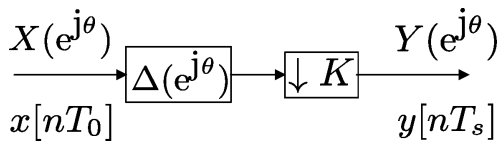

Fig. 5. Discrete-time model of one branch of nonuniform sampling scenario with $T_{s}=K \cdot T_{0}$ and discrete-time delay operator $\Delta\left(\mathrm{e}^{\mathrm{j} \theta}\right)$.

signal samples. With relative frequency $\theta$ and via the IFTD this results in

$$
y[n]=\frac{1}{2 \pi} \int_{\theta=-\pi}^{\pi} \mathrm{e}^{-\mathrm{j} \tau \theta} \mathrm{e}^{\mathrm{j} 2 n \theta} \mathrm{d} \theta=\frac{\sin (\tau \pi)}{(\tau-2 n) \pi}
$$

where the signal samples $y[n]$ denote the samples on the new time axis $n \cdot T_{s}=n \cdot 2[\mathrm{~s}]$.

On the other hand we can evaluate the same result by using (3). With $x[n]=\delta[n]$ we have $X\left(\mathrm{e}^{\mathrm{j} \theta}\right)=1$ and this equation reduces to

$$
Y\left(\mathrm{e}^{\mathrm{j} \theta}\right)=\frac{1}{2}\left\{\Delta\left(\mathrm{e}^{\mathrm{j} \theta / 2}\right)+\Delta\left(\mathrm{e}^{\mathrm{j} \theta / 2} \cdot \mathrm{e}^{\mathrm{j} \pi}\right)\right\} .
$$

We observe that the phase characteristic of the first term is a scaled version of the original phase characteristic of $\Delta\left(\mathrm{e}^{\mathrm{j} \theta}\right)$. The second term is first shifted over $\pi$ [rad] and then scaled. Because of the fact that the original phase contains a jump of $2 \tau \cdot \pi$ at the edge of the FI, the second term contains a phase jump at $\theta=0$. Thus

$$
\begin{aligned}
& \Phi\left\{\Delta\left(\mathrm{e}^{\mathrm{j} \theta / 2}\right)\right\}=-\tau \cdot \frac{\theta}{2}-\pi<\theta \leq \pi \\
& \Phi\left\{\Delta\left(\mathrm{e}^{\mathrm{j} \theta / 2} \cdot \mathrm{e}^{\mathrm{j} \pi}\right)\right\}= \begin{cases}-\tau \cdot\left(\frac{\theta}{2}+\pi\right) & -\pi<\theta \leq 0 \\
-\tau \cdot\left(\frac{\theta}{2}-\pi\right) & 0<\theta \leq \pi .\end{cases}
\end{aligned}
$$

Because of the phase jump at $\theta=0$, the output samples $y[n]$ have to be calculated by splitting the IFTD equation as follows:

$$
\begin{aligned}
y[n]= & \frac{1}{2 \pi}\left\{\int_{-\pi}^{0} \frac{1}{2}\left(\mathrm{e}^{-\mathrm{j} \tau \theta / 2}+\mathrm{e}^{-\mathrm{j} \tau(\theta / 2+\pi)}\right) \mathrm{e}^{\mathrm{j} n \theta} \mathrm{d} \theta\right. \\
& \left.\quad+\int_{0}^{\pi} \frac{1}{2}\left(\mathrm{e}^{-\mathrm{j} \tau \theta / 2}+\mathrm{e}^{-\mathrm{j} \tau(\theta / 2-\pi)}\right) \mathrm{e}^{\mathrm{j} n \theta} \mathrm{d} \theta\right\} \\
= & \frac{\sin (\tau \pi)}{(\tau-2 n) \pi} .
\end{aligned}
$$

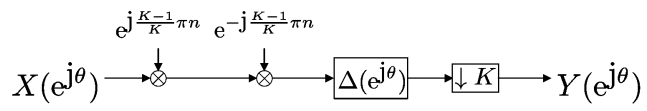

II

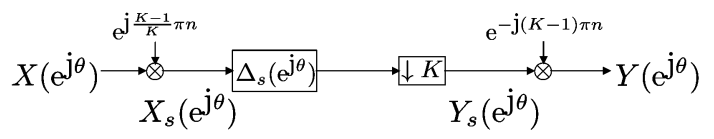

Fig. 6. Derivation of alternative discrete-time model of one branch by applying a modulation and demodulation operator.

A valid alternative model is obtained by shifting the frequency response of the delay operator before it is downsampled. This frequency shift, that can be performed in time domain by a modulation operator, is chosen in such a way that the phase jump does not appear in the FI of the aliased signal that results after the downsampling operation. This alternative model is sketched in Fig. 6. In order to start with the original discrete-time model, we have applied in the upper part of the figure a modulation operator followed by a demodulation operator. The result is that the spectrum of the input signal is first shifted over $((K-1) / K) \pi$ [rad] and then shifted over the same amount in the opposite direction. In the lower part of the figure, the demodulation operator is first moved over the delay $\Delta\left(\mathrm{e}^{\mathrm{j} \theta}\right)$, resulting in a delay from which the frequency response is given by

$$
\Delta_{s}\left(\mathrm{e}^{\mathrm{j} \theta}\right)=\Delta\left(\mathrm{e}^{\mathrm{j}(\theta-((K-1) / K) \pi)}\right) .
$$

Finally the demodulation operator is moved over the downsampling operator $\downarrow K$ which results in a demodulation operator of $\mathrm{e}^{-\mathrm{j}(K-1) \pi n}$.

The relation of the phase response between the original delay $\Delta\left(\mathrm{e}^{\mathrm{j} \theta}\right)$ and $\Delta_{s}\left(\mathrm{e}^{\mathrm{j} \theta}\right)$ is depicted in Fig. 7. In this figure, a new shifted Fundamental Interval $\left(\mathrm{FI}_{\mathrm{S}}\right)$ is defined as

$$
\mathrm{FI}_{\mathrm{s}}:-\left(\frac{1}{K}\right) \cdot \pi<\theta \leq\left(\frac{2 K-1}{K}\right) \cdot \pi
$$

In $\mathrm{FI}_{\mathrm{S}}$ the phase of $\Delta_{s}\left(\mathrm{e}^{\mathrm{j} \theta}\right)$ is defined by:

$$
\Delta_{s}\left(\mathrm{e}^{\mathrm{j} \theta}\right)=W_{K}^{-((K-1) / 2) \tau} \cdot \mathrm{e}^{-\mathrm{j} \tau \theta} .
$$




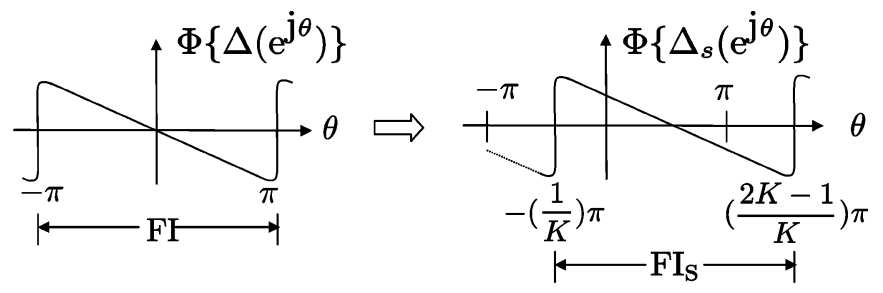

Fig. 7. Phase of original and shifted discrete-time delay.

Furthermore, by using the standard modulation and demodulation operators [5], we note that

$$
\begin{aligned}
X_{s}\left(\mathrm{e}^{\mathrm{j} \theta}\right) & =X\left(\mathrm{e}^{\mathrm{j} \theta} \cdot W_{K}^{(K-1) / 2}\right) \\
Y\left(\mathrm{e}^{\mathrm{j} \theta}\right) & =Y_{s}\left(\mathrm{e}^{\mathrm{j} \theta} \cdot \mathrm{e}^{\mathrm{j}(K-1) \pi}\right) .
\end{aligned}
$$

In a similar way as before, we can derive the following frequency domain expression for the discrete-time output samples of the alternative model of one branch:

$$
\begin{array}{r}
Y_{s}\left(\mathrm{e}^{\mathrm{j} \theta}\right)=\frac{1}{K} \sum_{p=0}^{K-1}\left(W_{K}^{(p-(K-1) / 2) \tau} \cdot \mathrm{e}^{-\mathrm{j} \tau \theta / K}\right) \\
\cdot X\left(\mathrm{e}^{\mathrm{j} \theta / K} \cdot W_{K}^{-(p-(K-1) / 2)}\right) .
\end{array}
$$

Substitution of $q=p-(K-1) / 2$ results in the following frequency domain expression that describes one branch of the alternative model of the recurrent nonuniform sampling scenario:

$$
Y_{s}\left(\mathrm{e}^{\mathrm{j} \theta}\right)=\frac{1}{K} \cdot \mathrm{e}^{-\mathrm{j} \tau \theta / K} \sum_{q=-(K-1) / 2}^{(K-1) / 2} W_{K}^{q \tau} \cdot X\left(\mathrm{e}^{\mathrm{j} \theta / K} W_{K}^{-q}\right)
$$

while the frequency response of the output is obtained by

$$
Y\left(\mathrm{e}^{\mathrm{j} \theta}\right)=Y_{s}\left(\mathrm{e}^{\mathrm{j} \theta} \cdot \mathrm{e}^{\mathrm{j}(K-1) \pi}\right) .
$$

Finally note that range of the running index $q$ of the summation in (14) is defined by

$$
q=-\frac{K-1}{2}: 1: \frac{K-1}{2}
$$

and, thus, $q$ needs not to be an integer.

Example 3: In this example, we will verify (14) by using the same parameters as in example 2. With these parameters (14) reduces to

$$
Y_{s}\left(\mathrm{e}^{\mathrm{j} \theta}\right)=\frac{1}{2} \mathrm{e}^{-\mathrm{j} \tau \theta / 2}\left\{\mathrm{e}^{\mathrm{j}(\pi / 2) \tau}+\mathrm{e}^{-\mathrm{j}(\pi / 2) \tau}\right\} .
$$

Applying the IFTD to $Y\left(\mathrm{e}^{\mathrm{j} \theta}\right)=Y_{s}\left(\mathrm{e}^{\mathrm{j} \theta} \cdot \mathrm{e}^{\mathrm{j} \pi}\right)$ results in the following equation:

$$
\begin{aligned}
y[n]= & \mathrm{e}^{-\mathrm{j} \pi n} \frac{1}{2 \pi} \int_{-\pi}^{\pi}\left\{\frac{1}{2} \mathrm{e}^{-\mathrm{j} \tau \theta / 2}\left(\mathrm{e}^{\mathrm{j}(\pi / 2) \tau}+\mathrm{e}^{-\mathrm{j}(\pi / 2) \tau}\right)\right\} \\
& \times \mathrm{e}^{\mathrm{j} n \theta} \mathrm{d} \theta \\
= & \frac{\sin (\tau \pi)}{(\tau-2 n) \pi}
\end{aligned}
$$

which is indeed the same result as in Example 2.

\section{B. Description of Alternative Discrete-Time Model}

By applying the modulation and demodulation operator, as described earlier, to each of the $K$ branches of the discrete-time model of the recurrent nonuniform sampling scenario we obtain the alternative discrete-time model as depicted in Fig. 8. Each of these branches can now be described as explained previously, which leads to the following set of equations for $k=$ $0,1, \ldots, K-1$ :

$$
Y_{s, k}\left(\mathrm{e}^{\mathrm{j} \theta}\right)=\frac{1}{K} \mathrm{e}^{-\mathrm{j} \tau_{k} \theta / K} \sum_{q=-(K-1) / 2}^{(K-1) / 2} W_{K}^{q \tau_{k}} X\left(\mathrm{e}^{\mathrm{j} \theta / K} W_{K}^{-q}\right) .
$$

We can combine this set of equations in the following vectormatrix notation:

$$
\underline{\mathbf{Y}}_{s}\left(\mathrm{e}^{\mathrm{j} \theta}\right)=\frac{1}{K} \cdot \Delta\left(\mathrm{e}^{\mathrm{j} \theta / K}\right) \cdot \mathbf{W} \cdot \underline{\mathbf{X}}\left(\mathrm{e}^{\mathrm{j} \theta / K}\right)
$$

with

$$
\begin{aligned}
& \underline{\mathbf{X}}\left(\mathrm{e}^{\mathrm{j} \theta / K}\right)=\left(X\left(\mathrm{e}^{\mathrm{j} \theta / K} W_{K}^{(K-1) / 2}\right), \ldots,\right. \\
&\left.X\left(\mathrm{e}^{\mathrm{j} \theta / K} W_{K}^{-(K-1) / 2}\right)\right)^{t} \\
& \mathbf{W}=\left(\underline{\mathbf{W}}_{K}^{\tau_{0}}, \ldots, \underline{\mathbf{W}}_{K}^{\tau_{k}}, \ldots, \underline{\mathbf{W}}_{K}^{\tau_{K}-1}\right)^{t} \\
& \underline{\mathbf{W}}_{K}^{\tau_{k}}=\left(W_{K}^{-((K-1) / 2) \cdot \tau_{k}}, \ldots, W_{K}^{((K-1) / 2) \cdot \tau_{k}}\right)^{t} \\
& \Delta\left(\mathrm{e}^{\mathrm{j} \theta / K}\right)=\operatorname{diag}\left\{\mathrm{e}^{-\mathrm{j} \tau_{0} \theta / K}, \ldots, \mathrm{e}^{-\mathrm{j} \tau_{K-1} \theta / K}\right\} \\
& \underline{\mathbf{Y}}_{s}\left(\mathrm{e}^{\mathrm{j} \theta}\right)=\left(Y_{s, 0}\left(\mathrm{e}^{\mathrm{j} \theta}\right), \ldots, Y_{s, K-1}\left(\mathrm{e}^{\mathrm{j} \theta}\right)\right)^{t} .
\end{aligned}
$$

Finally a demodulation operator is applied to each of the $K$ branches, which is expressed in the following vector:

$$
\begin{aligned}
& \underline{\mathbf{Y}}\left(\mathrm{e}^{\mathrm{j} \theta}\right) \\
& =\left(Y_{s, 0}\left(\mathrm{e}^{\mathrm{j} \theta} \cdot \mathrm{e}^{\mathrm{j}(K-1) \pi}\right), \ldots, Y_{s, K-1}\left(\mathrm{e}^{\mathrm{j} \theta} \cdot \mathrm{e}^{\mathrm{j}(K-1) \pi}\right)\right)^{t} .
\end{aligned}
$$

Note that a nonuniform DFT matrix has been defined in [2]. In a similar way, the matrix $\mathbf{W}$ can be viewed as a nonuniform DFT matrix.

From (17) and (19), it follows that the description of the alternative discrete-time model of the recursive nonuniform sampling scenario consists of a mixture of $K$ uniformly distributed downsampled frequency bands $X\left(\mathrm{e}^{\mathrm{j} \theta / K} \cdot W_{K}^{(K-1) / 2}\right), \ldots, X\left(\mathrm{e}^{\mathrm{j} \theta / K} \cdot W_{K}^{-(K-1) / 2}\right)$.

\section{DFT MOdUlated Filterbank REPRESENTATION OF Alternative Discrete-Time Model}

From literature, it is well known that a set of $K$ uniformly distributed downsampled frequency bands can be obtained by the analysis part of a DFT modulated filterbank representation [13]. In our case, however, we have to modify this representation because of the fact that the input signal samples of the alternative discrete-time model are first modulated. For this reason, we will first give in this section a short derivation of the analysis part of a DFT modulated filterbank from which the input signal samples are first modulated. In the alternative model, these resulting frequency bands, of vector $\underline{\mathbf{X}}\left(\mathrm{e}^{\mathrm{j} \theta / K}\right)$, are mixed by the 


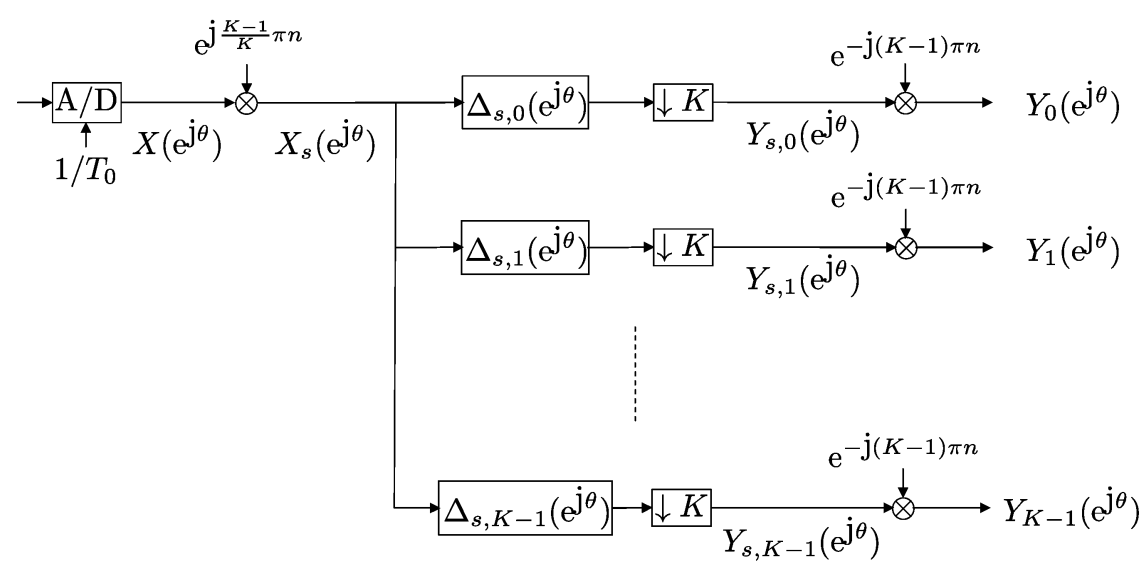

Fig. 8. Alternative discrete-time model of recurrent nonuniform sampling scenario by applying modulation and demodulation operators.

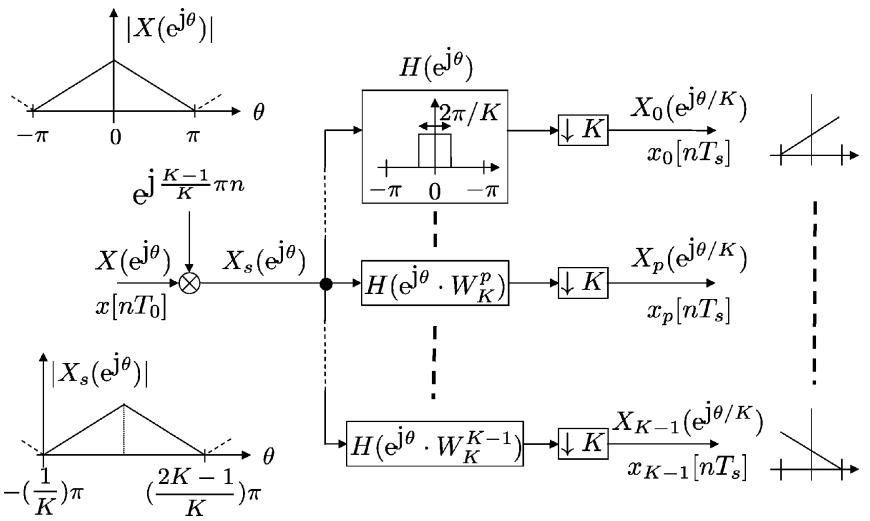

Fig. 9. Basic structure of uniform filterbank from which the input signal samples are first modulated.

nonuniform DFT matrix $\mathbf{W}$ and than filtered by the diagonal filter matrix $\Delta\left(\mathrm{e}^{\mathrm{j} \theta / K}\right)$. In this way, we obtain a DFT modulated filterbank representation of the alternative discrete-time model of the recursive nonuniform sampling scenario.

For this purpose, we start with the basic structure of a uniform filterbank of which the input signal samples are first modulated, which is depicted in Fig. 9. At the top LHS of this figure, we have drawn a triangular shape, denoting the amplitude characteristic $\left|X\left(\mathrm{e}^{\mathrm{j} \theta}\right)\right|$ of the input signal samples $x\left[n T_{0}\right]$. The input signal samples are first modulated, which results in a shifted input signal spectrum $X_{s}\left(\mathrm{e}^{\mathrm{j} \theta}\right)$. The shifted amplitude characteristic is shown at the bottom LHS of the figure. This spectrum of the modulated input signal is split into $K$ uniform frequency bands of width $2 \pi / K$ [rad] by using $K$ parallel filters $H\left(\mathrm{e}^{\mathrm{j} \theta} \cdot W_{K}^{p}\right)$, for $p=0,1, \ldots, K-1$. These filters are frequency shifted versions of a prototype filter, which is a lowpass filter (LPF) $H\left(\mathrm{e}^{\mathrm{j} \theta}\right)$, with cut-off frequency $\left|\theta_{c}\right|=\pi / K$. Each of the filtered signals are downsampled by a factor of $K$, resulting in a set of $K$ parallel discrete-time signals $x_{p}\left[n T_{s}\right]$ for $p=0,1, \ldots, K-1$ denoting the time domain representation of the downsampled frequency bands

$$
X_{p}\left(\mathrm{e}^{\mathrm{j} \theta}\right)=\frac{1}{K} X\left(\mathrm{e}^{\mathrm{j} \theta / K} \cdot W_{K}^{-(p-(K-1) / 2)}\right) .
$$

It is well known from literature that a DFT modulated filterbank is an efficient way for the realization of this uniform filterbank
[13]. This efficient structure uses the following polyphase decomposition for the prototype LPF $H\left(\mathrm{e}^{\mathrm{j} \theta}\right)$ :

$$
H\left(\mathrm{e}^{\mathrm{j} \theta}\right)=\sum_{t=0}^{K-1} \mathrm{e}^{-\mathrm{j} t \theta} H_{t}^{K}\left(\mathrm{e}^{\mathrm{j} K \theta}\right) .
$$

The DFT modulated filterbank representation is obtained by using the polyphase decomposition for each of the filters $H\left(\mathrm{e}^{\mathrm{j} \theta} \cdot W_{K}^{p}\right)$ and then "moving" the downsamplers over these frequency shifted and polyphase decomposed filters. This results in a delay line in which $K$ successive samples of the input signal are collected. This delay line is followed by a set of $K$ parallel downsamplers and polyphase filters $H_{p}^{K}\left(\mathrm{e}^{\mathrm{j} \theta}\right)$. The frequency-shifted versions of the prototype filter are obtained by using a DFT. Furthermore, we will assume in our development an ideal LPF prototype filter $H\left(\mathrm{e}^{\mathrm{j} \theta}\right)$. For this ideal case, it can be shown that the polyphase filters $H_{t}^{K}\left(\mathrm{e}^{\mathrm{j} \theta}\right)$ are all-pass and represent the following set of (noncausal) fractional delays [5]:

$$
H_{t}^{K}\left(\mathrm{e}^{\mathrm{j} \theta}\right)=\mathrm{e}^{\mathrm{j} t \theta / K}
$$

As pointed out before, in our development we first modulate the input signal samples. By "moving" this modulation operator $\mathrm{e}^{\mathrm{j}((K-1) / K) \pi n}$ over the delay line and downsamplers, we obtain the analysis part of a DFT modulated filterbank representation from which the input signal samples are first modulated, as depicted in Fig. 10. At the LHS of this figure, we see the delay line, that consists of $K-1$ (causal, uniform) delay elements of $T_{0}$ [s]. These delay elements are denoted by the symbol $\mathrm{e}^{-\mathrm{j} \theta}$. At the RHS, we used the matrix $\mathbf{F}^{-1}$, which is the inverse of the DFT matrix $\mathbf{F}$ from which the $p, q$ th element is defined as $(\mathbf{F})_{p, q}=W_{K}^{p \cdot q}$. The (noncausal) fractional delays, that represent the ideal polyphase filters of (23), can be combined in the diagonal filter matrix $\mathbf{D}^{-1}\left(\mathrm{e}^{\mathrm{j} \theta / K}\right)$, which is the inverse of

$$
\mathbf{D}\left(\mathrm{e}^{\mathrm{j} \theta / K}\right)=\operatorname{diag}\left\{\mathrm{e}^{-\mathrm{j} 0 \cdot \theta / K}, \ldots, \mathrm{e}^{-\mathrm{j}(K-1) \cdot \theta / K}\right\} .
$$

The "move" of the modulation operator $\mathrm{e}^{\mathrm{j}((K-1 / K) \pi n}$ over the delay line and downsamplers results in a set of $K$ modulators $\mathrm{e}^{\mathrm{j}(K-1) \pi n}$ and the matrix $\mathbf{S}^{-1}$, which is the inverse of the following frequency shift matrix $\mathbf{S}$ :

$$
\mathbf{S}=\operatorname{diag}\left\{W_{K}^{-((K-1) / 2) \cdot 0}, \ldots, W_{K}^{-((K-1) / 2) \cdot(K-1)}\right\} .
$$




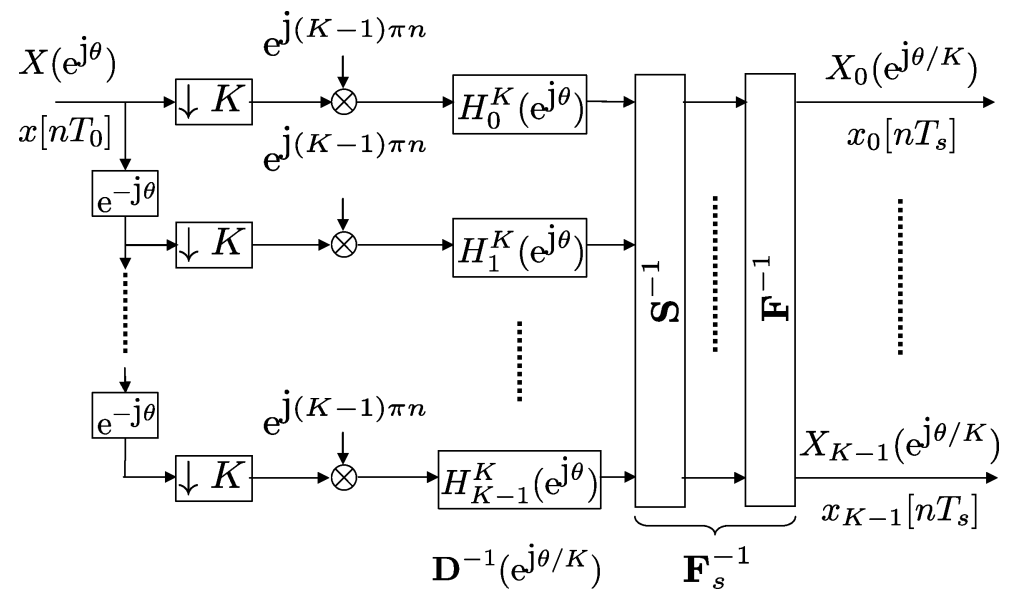

Fig. 10. Structure of uniform DFT modulated filterbank from which the input signal samples are first modulated.

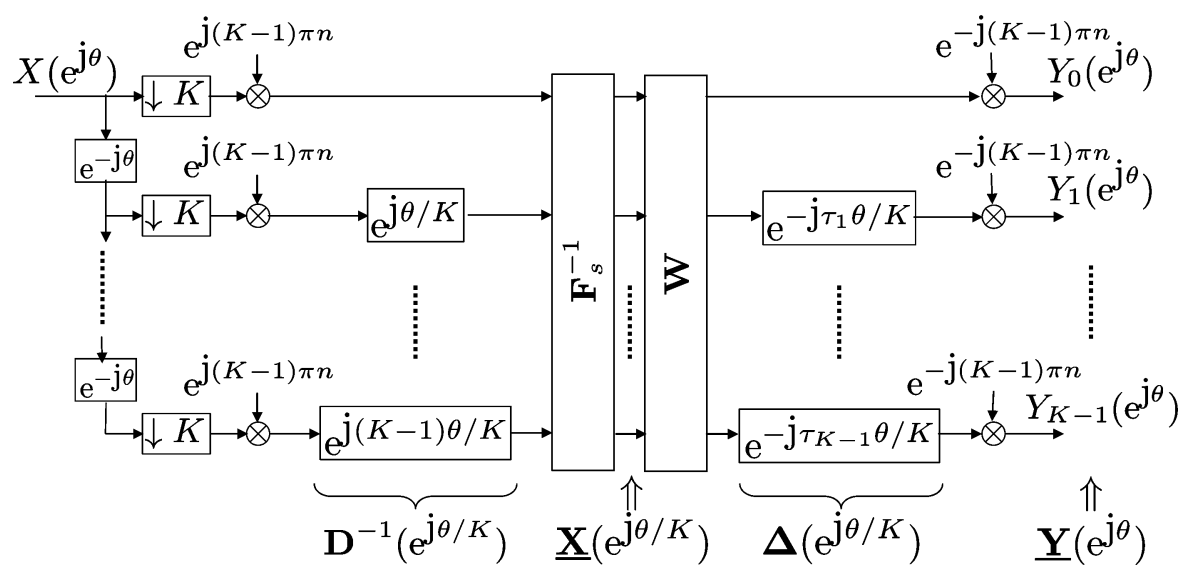

Fig. 11. DFT modulated filterbank representation of the alternative discrete-time model of the recursive nonuniform sampling scenario.

Combining this frequency shift matrix $\mathbf{S}$ with the DFT matrix $\mathbf{F}$ results in a (shifted) DFT matrix $\mathbf{F}_{s}$ that is defined as follows:

$$
\begin{aligned}
\mathbf{F}_{s} & =\mathbf{S} \cdot \mathbf{F}=\left(\underline{\mathbf{W}}_{K}^{0}, \ldots, \underline{\mathbf{W}}_{K}^{k}, \ldots, \underline{\mathbf{W}}_{K}^{K-1}\right)^{t} \\
\underline{\mathbf{W}}_{K}^{k} & =\left(W_{K}^{-((K-1) / 2) \cdot k}, \ldots, W_{K}^{((K-1) / 2) \cdot k}\right)^{t} .
\end{aligned}
$$

Note that this DFT matrix $\mathbf{F}_{s}$ and the nonuniform DFT matrix $\mathbf{W}$, as defined in (19), have the same structure. From this structure it follows easily that in case of uniformly distributed delays, thus if $\tau_{k}=k$, we obtain $\mathbf{W}=\mathbf{F}_{s}$.

Combining all these results and using (18), we arrive at a DFT modulated filterbank representation of the alternative discrete-time model of the recursive nonuniform sampling scenario, which is depicted in Fig. 11. Thus by applying an appropriate modulation and demodulation operator, the relationship between the recurrent nonuniform and uniform sampling schemes can be described by the analysis part of a uniform DFT modulated filterbank from which the $K$ uniformly distributed and downsampled frequency bands are first mixed with the nonuniform DFT matrix $\mathbf{W}$. Then each of the resulting branches is time aligned by a delay $\mathrm{e}^{-\mathrm{j} \tau_{k} \theta / K}$.
Finally, it is noted here that the modulation/ demodulation operators $\mathrm{e}^{ \pm \mathrm{j}(K-1) \pi n}$ in this figure simply reduce to

$$
\mathrm{e}^{ \pm \mathrm{j}(K-1) \pi n}= \begin{cases}1 & \text { for odd } K \\ (-1)^{n} & \text { foreven } K\end{cases}
$$

For odd $K$, the phase jump is automatically at the edge of the FI of the aliased signal that results after the downsampling operator and no frequency shift is needed. For even $K$ however, the phase jump is exact in the middle of the aliased signal (verify, e.g., Example 2) that results after the downsampling operator and a frequency shift of $\pi$ [rad] is needed.

\section{EFFicient Dft Modulated Filterbank Realization OF RECONSTRUCTION}

An important side benefit of the filterbank description of the alternative model is that we can now simply derive an efficient reconstruction structure. In order to reconstruct the original signal samples $x\left[n T_{0}\right]$, we first give the inverse of (18), which results in

$$
\frac{1}{K} \cdot \underline{\mathbf{X}}\left(\mathrm{e}^{\mathrm{j} \theta / K}\right)=\mathbf{W}^{-1} \cdot \Delta^{-1}\left(\mathrm{e}^{\mathrm{j} \theta / K}\right) \cdot \underline{\mathbf{Y}}_{s}\left(\mathrm{e}^{\mathrm{j} \theta}\right) .
$$




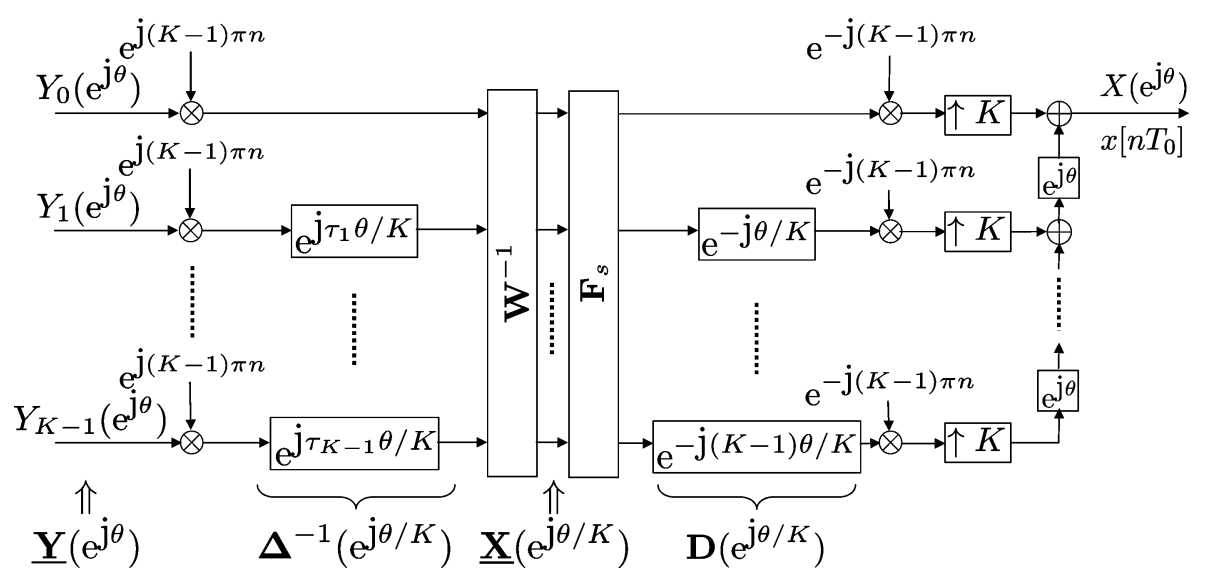

Fig. 12. Efficient DFT modulated filterbank realization of reconstruction of uniform signal samples from recursive nonuniform signal samples.

Furthermore, it is known from [13] that the DFT modulated synthesis filterbank is an efficient way to reconstruct a signal from its uniform frequency bands, available in vector $\underline{\mathbf{X}}\left(\mathrm{e}^{\mathrm{j} \theta / K}\right)$. This is depicted in the RHS of Fig. 12. This figure represents the synthesis part of an efficient DFT modulated filterbank realization with which we can reconstruct uniform discrete-time signal samples $x\left[n T_{0}\right]$ from recurrent nonuniform discrete-time signal samples $y_{0}\left[n T_{s}\right], \ldots, y_{K-1}\left[n T_{s}\right]$. The first step of this reconstruction scheme is the modulation of the incoming signals. This modulation is needed to obtain downsampled signals that do not have a phase jump in the FI. In order to further process the $K$ parallel signals, a proper time alignment is needed. This time alignment is taken care of by the inverse of the diagonal matrix $\Delta\left(\mathrm{e}^{\mathrm{j} \theta / K}\right)$. Each of the resulting signals contains a mixture of $K$ uniform filterbands $X_{p}\left(\mathrm{e}^{\mathrm{j} \theta}\right)$ for $p=0,1, \ldots, K-1$ as defined in (21). This mixture is demixed by the inverse of matrix $\mathbf{W}$. From this point onwards the structure is equivalent to the synthesis part of an efficient DFT modulated uniform filterbank, which is the inverse of the analysis structure as depicted in Fig. 10. For this reason, this reconstruction structure can be viewed as a natural extension of the synthesis part of a uniform DFT modulated filterbank.

Finally, we note here that in case of uniform sampling, thus for $\tau_{k}=k$, we have $\mathbf{F}_{s} \cdot \mathbf{W}^{-1}=\mathbf{I}$, with $\mathbf{I}$ the identity matrix. Furthermore, if we assume that $H\left(\mathrm{e}^{\mathrm{j} \theta}\right)$ is an ideal LPF, we also have $\mathbf{D}\left(\mathrm{e}^{\mathrm{j} \theta}\right) \cdot \Delta^{-1}\left(\mathrm{e}^{\mathrm{j} \theta}\right)=\mathbf{I}$. For this specific situation, the whole reconstruction scheme of Fig. 12 reduces, as expected, to a time-interleaved structure which consists of a set of $K$ parallel upsamplers and (noncausal) delays.

\section{Simulation Results}

In order to verify the results we implemented, a causal Matlab version of the ideal noncausal structure as depicted in Fig. 12 and compared the original input signal $x$ to the causal reconstructed signal $\hat{x}$. Causality of the reconstruction structure is obtained by applying the following two delay operators.

1) Since $0 \leq \tau_{k}<K$, an extra (causal) delay of $T_{s}=K \cdot T_{0}$ [s] is added to each of the noncausal delays $\mathrm{e}^{\mathrm{j} \tau_{k} \theta / K}$ of $\Delta^{-1}$.

2) The transpose of the delay line at the RHS of Fig. 12 is used, resulting in a delay of $(K-1) \cdot T_{0}[\mathrm{~s}]$.
TABLE I

\begin{tabular}{|l||c|c|c|c|}
\hline Distribution of delays & $\tau_{0}$ & $\tau_{1}$ & $\tau_{2}$ & $\tau_{3}$ \\
\hline \hline a) Uniform & 0 & 1 & 2 & 3 \\
\hline b) Near uniform & 0 & 0.95 & 2.05 & 3.05 \\
\hline c) Strong nonuniform & 0 & 0.5 & 0.95 & 3.55 \\
\hline
\end{tabular}

There are many different ways to cope with the implementation of noninteger delays [11], [13]. In our implementation, we choose the following approach: A noninteger delay can be approximated by a fractional delay. With $L$ an integer number, a fractional delay with resolution $1 / L$ can be implemented very efficiently by using the polyphase decomposition of an FIR LPF with cut-off frequency $\left|\theta_{c}\right|=\pi / L$. In our implementation, we used a resolution of $1 / L=1 / 20=0.05$. The length of the used fractional delay FIR filters was $L_{p}=74$ coefficients. Furthermore, we used two different options for the reconstruction, namely, the following:

1) "reconstruct": Filterbank reconstruction as discussed in this paper;

2) "time interleave": Reconstruction by simple uniform timeinterleaving the signal samples $y_{p}\left[n T_{s}\right]$, thus, only using the upsamplers $\uparrow K$ and the delay line. Obviously this option is only perfect when the delays $\tau_{k}$ are uniformly distributed and the results will show that small deviations from this uniform distribution already cause significant errors.

Finally, we used $K=4, T_{0}=1$ and the input signal samples are generated as

$$
x[n]=\sin (0.1 \pi \cdot n)+2 \sin (0.75 \pi \cdot n) .
$$

We have run three different simulations, each with 2048 signal samples. The first one with uniformly distributed delays, the second one with nearly uniformly distributed delays (within 5\% deviation from uniform) and in the third simulation we used strongly nonuniformly distributed delays. The exact values of the delays are given in Table I. 

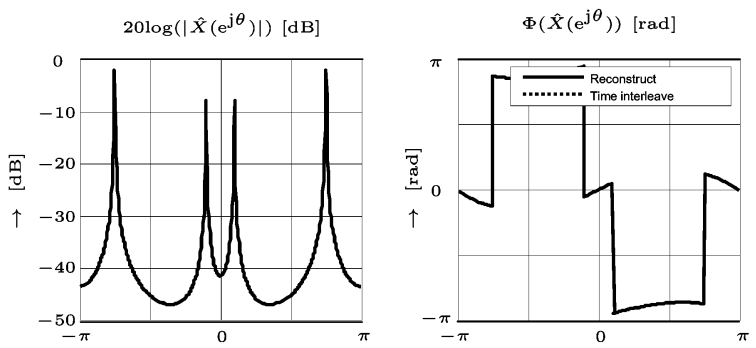

a) Uniformly distributed delays $\rightarrow \theta[\mathrm{rad}]$
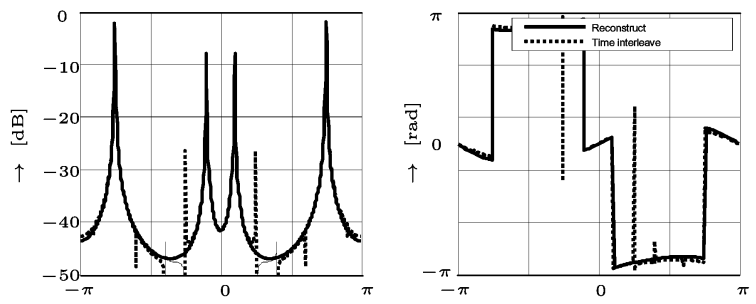

b) Nearly uniformly distributed delays $(<5 \%) \rightarrow \theta[\mathrm{rad}]$
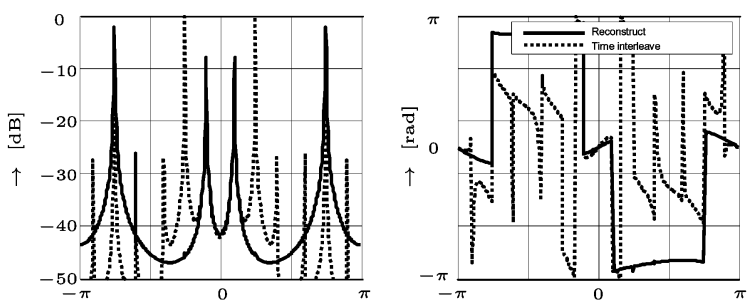

c) Strongly nonuniformly distributed delays $\rightarrow \theta[\mathrm{rad}]$

Fig. 13. Simulation results for different distribution of delays.

The simulation results are depicted in Fig. 13. For each set of delays the figure shows for the two reconstruction options "Reconstruct" (solid line) and "Time interleave" (dotted line) at the LHS the amplitude characteristic $20 \log \left(\left|\hat{X}\left(\mathrm{e}^{\mathrm{j} \theta}\right)\right|\right)[\mathrm{dB}]$ and at the RHS the phase characteristic $\Phi\left\{\hat{X}\left(\mathrm{e}^{\mathrm{j} \theta}\right)\right\}$ [rad] of the reconstructed signal samples $\hat{x}[n]$, both as a function of the frequency $\theta$ with $-\pi \leq \theta<\pi$. To make these plots we used one fragment of 512 samples of $\hat{x}[n]$ and applied an FFT of the length 512 to these (unwindowed) data samples. Furthermore, we compared the samples of the chosen fragment with the corresponding original signal samples of $x[n]$ and calculated the following error: $\epsilon=(1 / 512) \sum_{n \in \text { fragment }}|x[n]-\hat{x}[n]|$.

In the first simulation result, a) uniformly distributed delays, the plots show no difference between the "Time interleave" and the "Reconstruct" option. The reason is that in this case the "Time interleave" option is perfect, while the "Reconstruct" option has a very small error, $\epsilon_{a}=2.82 \cdot 10^{-4}$. This small error results from the imperfections of the used filters. This error $\epsilon_{a}$ can be used as a reference for the other simulation results. In the second simulation result, b) nearly uniformly distributed delays, the error of the "Reconstruct" option is still very small, $\epsilon_{b}=3.16 \cdot 10^{-4}$, while the results of the "Time interleave" option already show some erroneous peaks. These peaks are also described in [8] and [9]. Finally, the third simulation result, c) strongly nonuniformly distributed delays, shows that the "Reconstruct" option is still very good with error $\epsilon_{c}=8.91 \cdot 10^{-4}$, while the results of the "Time interleave" option become extremely bad.

\section{CONCLUSION AND Future RESEARCH}

In this paper, we clarified the relationship between uniform and recurrent nonuniform sampling schemes. This insight was obtained from a new alternative discrete-time model of the nonuniform recurrent sampling scenario. It was shown that this model could be described by the analysis part of a uniform DFT modulated filterbank from which the $K$ uniformly distributed and downsampled frequency bands are mixed by the nonuniform DFT matrix W. A side benefit of this new model was the introduction of an efficient structure with which one can reconstruct uniform discrete-time Nyquist signal samples from recurrent nonuniform samples with known mutual delays between the nonuniform distributed samples. This reconstruction structure can be viewed as a natural extension of the synthesis part of an uniform DFT modulated filterbank.

For real practical applications, the derived filterbank structure has the following limitations, which are relevant topics for future research:

1) The used filterbank is critically sampled, thus the number of frequency bands, say $M$, and the downsample factor $K$ are equal $(M=K)$. In practice, the filters are nonideal and have a transition band, which causes leakage from one frequency band to another. In such a case, it is better to use noncritical (over)sampled filterbanks [13], for which $M>K$.

2) The delays are assumed to be known. In many practical situations, this is not the case. An adaptive mechanism, similar to the ones as derived in [8], [12], and [19], is needed to make an online estimation of the delays.

3 ) In practice, different delays can be (almost) equal. The reconstruction (28) makes use of the inverse of the nonuniform DFT matrix $\mathbf{W}$. This inverse matrix will become numerically unstable if the delays $\tau_{p}$ and $\tau_{q}$ are almost equal for $p \neq q$.

\section{ACKNOWLEDGMENT}

The authors would like to thank F. Bruekers and J. van de Laar for their valuable comments.

\section{REFERENCES}

[1] A. Aldroubi and K. Gröchenig, "Non-uniform sampling and reconstruction in shift-invariant spaces," SIAM Rev., vol. 43, pp. 585-620, 2001.

[2] B. Sonali and K. M. Sanjit, The Nonuniform Discrete Fourier Transform and Its Applications in Signal Processing. London, U.K.: Kluwer Academic.

[3] F. C. Kwan, "A multidimensional extension of Papoulis' generalized sampling expansion with the application in minimum density sampling," in In Advanced Topics in Shannon Sampling and Interpolation Theory. New York: Springer-Verlag, 1993.

[4] Y. C. Eldar and A. V. Oppenheim, "Filter reconstruction of bandlimited signals from nonuniform generalized samples," IEEE Trans. Signal Process., vol. 48, no. 10, pp. 2864-2875, Oct. 2000.

[5] A. W. M. van den Enden, Efficiency in Multirate and Complex Digital Signal Processing. New York: Delta, 2001.

[6] C. Herley and P. W. Wong, "Minimum rate sampling and reconstruction of signals with arbitrary frequency support," IEEE Trans. Inf. Theory, vol. 45, no. 5, pp. 1555-1564, Jul. 1999.

[7] S. H. Izen, "Generalized sampling expansion on lattices," IEEE Trans. Signal Process., vol. 53, no. 6, pp. 1949-1963, Jun. 2005.

[8] S. M. Jamal, D. Fu, M. P. Singh, P. J. Hurst, and S. H. Lewis, "Calibration of sample-time error in two-channel time-interleaved analog-toDigital Convertor," IEEE Trans. Circuits Syst. I, Reg. Papers, vol. 51, no. 1, pp. 130-139, Jan. 2004. 
[9] Y.-C. Jenq, "Perfect reconstruction of digital spectrum from nonuniform sampled signals," IEEE Trans. Instrum. Meas., vol. 46, pp. 649-652, Jun. 1997.

[10] H. Johansson and P. Löwenborg, "Reconstruction of nonuniformly sampled bandlimited signals by means of time-varying discrete-time FIR filters," EURASIP J. Appl. Signal Process., vol. 2006, pp. 1-18.

[11] V. Välimäki, M. Karjalainen, and U. Laine, "Splitting the unit delay," IEEE Signal Process. Mag., pp. 30-60, Jan. 1996.

[12] P. Marziliano and M. Vetterli, "Reconstruction of irregularly sampled discrete-time bandlimited signals with unknown sampling locations," IEEE Trans. Signal Process., vol. 48, no. 12, pp. 3462-3471, Dec. 2000.

[13] K. M. Sanjit, Digital Signal Processing; A Computer Based Approach. New York: McGraw-Hill Int..

[14] A. Papoulis, "Generalized sampling expansion," IEEE Trans. Circuits Syst., vol. CAS-24, pp. 652-654, Nov. 1977.

[15] T. Strohmer, "Computationally attractive reconstruction of bandlimited images for irregular samples," IEEE Trans. Image Process., vol. 6, no. 4, pp. 540-548, Apr. 1997.

[16] M. Unser and A. Aldroubi, "A general sampling theory for nonideal acquisition devices," IEEE Trans. Signal Process., vol. 42, no. 11, pp. 2915-2925, Nov. 1994.

[17] M. Unser and J. Zerubia, "Generalized sampling without bandlimited constraints," in Proc. ICASSP, 1997, vol. 3, pp. 2113-2116.

[18] P. P. Vaidyanathan, "Generalizations of the sampling theorem: Seven decades after Nyquist," IEEE Trans. Circuits Syst. I, Fund. Theory Appl., vol. 48, no. 9, pp. 1094-1109, Sep. 2001.

[19] P. Vandewalle, L. Sbaiz, J. Vandewalle, and M. Vetterli, "Super-resolution from unregistered and totally aliased signals using subspace methods," IEEE Trans. Signal Process., vol. 55, no. 7, pp. 3687-3703, Jul. 2007.

[20] R. Venkataramani and Y. Bresler, "Perfect reconstruction formulas and bounds on aliasing error in sub-Nyquist nonuniform sampling multiband signals," IEEE Trans. Inf. Theory, vol. 46, no. 6, pp. 2173-2183, Sep. 2000.

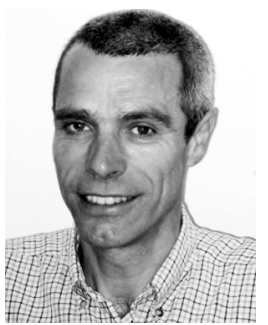

Piet Sommen received the Ingenieur degree in electrical engineering from Delft University of Technology, The Netherlands, in 1981 and the Ph.D. degree in 1992 from Eindhoven University of Technology, Eindhoven, The Netherlands.

From 1981 until 1989, he was with Philips Research Laboratories, Eindhoven. There, he was first engaged in research on $\mathrm{CAD}$ for circuit design, while later he worked in a radio and data transmission group where his main interest was on adaptive signal processing. Since 1989, he has been on the faculty of electrical engineering from Eindhoven University of Technology, where he is currently an Associate Professor. He is involved with different university courses and education projects all in the area of signal processing. His main field of interest is signal processing, with the emphasis on research and applications of adaptive array signal processing.

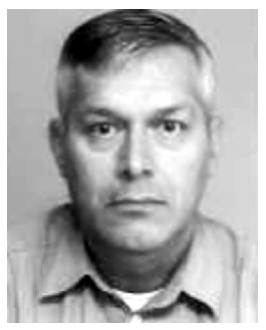

Kees Janse received the B.S. degree in electrical engineering in 1976 from the Polytechnical School, Vlissingen, The Netherlands.

He joined the acoustics group of Philips Research Laboratories in 1978, where his work focused on measuring techniques and signal analysis, for example, evaluating loudspeakers with the aid of the Wigner distribution. In 1984, he joined the Project Centre of Philips Research, where his work focused on the design of geographic data bases for car navigation systems. In 1988, he joined the Radio Data Transmission Group (and later on the Digital Signal Processing Group) of Philips Research and has since worked in the field of hands-free signal processing, including acoustic echo cancellation, acoustic feedback suppression, acoustic noise reduction, and dereverberation of speech signals. He has written and presented papers and holds a number of patents in his field. 